\title{
Non-universality of chaotic classical dynamics: implications for quantum chaos
}

\author{
M. Wilkinson ${ }^{1}$ and B. Mehlig ${ }^{2}$ \\ ${ }^{1}$ Department of Physics and Applied Physics, University of Strathclyde, Glasgow, G4 ONG, Scotland, U.K. \\ ${ }^{2}$ Theoretical Physics, University of Oxford, Oxford, OX1 3NP, England, U.K.
}

(August 10, 2006)

\begin{abstract}
It might be anticipated that there is statistical universality in the long-time classical dynamics of chaotic systems, corresponding to the universal correspondence of their quantum spectral statistics with random matrix models. We argue that no such universality exists. We discuss various statistical properties of long period orbits: the distribution of the phase-space density of periodic orbits of fixed length and a correlation function of periodic-orbit actions, corresponding to the universal quantum spectral two-point correlation function. We show that bifurcations are a mechanism for correlations of periodic-orbit actions. They lead to a result which is non-universal, and which in general may not be an analytic function of the action difference.
\end{abstract}

It has been appreciated for many years that sufficiently complex quantum systems exhibit a high degree of universality [1]: many statistical properties of their spectra usually fall into one of three classes, exemplified by the three Gaussian random matrix ensembles introduced by Dyson [2]. It is natural to ask whether an analogous degree of universality exists in classical dynamics, and if it exists whether it underlies the universality observed in the behaviour of quantum systems. This paper suggests what the appropriate classical analog of quantum spectral universality should be, and gives arguments supporting the view that there is, in general, no classical universality underlying that of quantum systems.

The universality exhibited by spectral properties is confined to statistics which are sufficiently short ranged in energy. This implies that the universal features are associated with dynamics over long time scales: they may be associated with universal properties of the long-time classical dynamics, or they may be purely quantum. It is natural to anticipate that universal behaviour, if present at all, will only be manifest in properties which characterise small regions of phase space, since the large scale structure of phase space can clearly be non-universal.

An example of a property which could show universality is the statistical characterisation of the distribution of points periodic under the $N^{\text {th }}$ iterate of a chaotic area preserving maps of the form $\alpha_{n+1}=\mathcal{M}\left(\alpha_{n}\right)$, with $\alpha=(x, p)$. These points are illustrated in Fig. 1 for the case of the $7^{\text {th }}$ iterate of the standard map [3]

$$
\begin{aligned}
& x_{n+1}=x_{n}+p_{n}, \\
& p_{n+1}=p_{n}+K \sin \left(x_{n+1}\right)
\end{aligned}
$$

with $K=6$ and the $11^{\text {th }}$ iterate of a modified cat map

$$
\left(\begin{array}{l}
x_{n+1} \\
p_{n+1}
\end{array}\right)=\left(\begin{array}{ll}
1 & 1 \\
1 & 2
\end{array}\right)\left(\begin{array}{l}
x_{n} \\
p_{n}
\end{array}\right)+K\left(\begin{array}{l}
u_{n} \\
0
\end{array}\right)
$$

with $u_{n}=\sin \left(x_{n}+2 p_{n}\right)$ and $K=2$. The patterns displayed in Fig. 1 are complex and show very wide fluctuations in the density of points. They are clearly different, but local statistical properties of their fine-scale structure might be equivalent after scaling the coordinates to give the same mean density of points.

Gutzwiller's trace formula [4] gives a relation between classical periodic orbits and quantum spectra. The formula is exact for a small number of special systems, but it cannot be exact in general because it contains no information about the choice of quantisation procedure [5]. In [6,7] Gutzwiller's formula is combined with the observation that spectral correlations are described by random matrix theory to infer that the actions of long period orbits are correlated. The correlations are a function of the action difference which was predicted to be universal within each of Dyson's symmetry classes, and an analytic form was quoted for the GUE ensemble. In systems where Gutzwiller's trace formula is exact (for a discussion of the three known examples see [4, 12]) such classcial correlations must certainly exist. However, in general the trace formula is not exact and it is thus necessary to find an entirely classical mechanism for such correlations. Up to now no such mechanism has been found.

The paper is organized as follows. We will first argue that the long-time, local structure of phase-space of chaotic systems is non-universal, using theoretical arguments and numerical experiments. We then characterize the remarkably strong fluctuations of the density of periodic points in Fig. 1 and provide theoretical arguments as well as numerical evidence that these fluctuations are log-normal. Finally we propose an entirely classical mechanism for periodic-orbit correlations, based on the statistical properties of bifurcations in the long-time dynamics. This mechanism gives rise to a non-universal and possibly non-analytic contribution to the correlation function.

We will first show that the long-time, local structure of phase space is not universal. Our argument is based upon considering a particular statistic: we will consider the proportion $P(N)$ of trajectories which are elliptic upon $N$ iterations of area-preserving maps such as (1), (2). The results generalise directly to continuous time 
systems. The stability of a point periodic under $N$ iterations of $\mathcal{M}, \alpha=\mathcal{M}^{N}(\alpha)$, is described by the monodromy matrix $M_{N}(\alpha)=\partial \mathcal{M}^{N}(\alpha) / \partial \alpha$. The point $\alpha=\mathcal{M}(\alpha)$ is elliptic (hyperbolic) if $\left|\operatorname{tr} M_{N}\right|<2\left(\left|\operatorname{tr} M_{N}\right|>2\right)$. The terms elliptic and hyperbolic will be used in the same way to describe any trajectory, regardless of whether it is closed.

For chaotic maps, in the large- $N$ limit the elliptic and hyperbolic trajectories are finely intermingled, implying that $P(N)$ is independent of any smooth scaling of the phase-space coordinates, and $P(N)$ will decrease rapidly with increasing $N$. Consider the distribution of values of $\operatorname{tr} M_{N}(\alpha)$ for large values of $N$. The monodromy matrix $M_{N}$ is a product of elementary monodromy matrices $m(\alpha)=M_{1}(\alpha)$ for individual applications of the mapping $M_{N}(\alpha)=\prod_{n=1}^{N} m\left(\alpha_{n}\right)$ where $\alpha_{n}$ is the phase-space point reached from $\alpha$ after $n$ applications of the mapping. The typical values of the elements of the matrix $M_{N}$ are expected to grow exponentially, in the sense that the mean of the logarithm of some norm of the matrix $M_{N}$ should grow linearly with time: $\lambda=\frac{1}{2} \lim _{N \rightarrow \infty} N^{-1}\left\langle\log \operatorname{tr}\left[M_{N}^{T}(\alpha) M_{N}(\alpha)\right]\right\rangle_{\alpha}$ is termed the Lyapunov exponent. This suggests that elliptic trajectories are rare in the long-time limit, and that if a universal form exists for the function $P(N)$, it might be expected to be exponential,

$$
P(N) \sim A \exp (-a \lambda N)
$$

for $N \gg 1$, where $A$ and $a$ are universal constants.

Determing the fraction of elliptic trajectories is equivalent to determining the probability that $\operatorname{tr} M_{N}$ lies in the interval $[-2,2]$. Because the typical value of $\operatorname{tr} M_{N}$ is exponentially large, this question relates to the tail of the distribution. In the case of a chaotic map the succesive positions $\alpha_{n}$ have the characteristics of random numbers, and the monodromy matrix $M_{N}$ may be modelled as a product of random matrices. A product of a large number of random scalars has a log-normal distribution, and it is therefore natural to expect that $\operatorname{tr} M_{N}$ will have a log-normal distribution. The central limit theorem is only applicable sufficiently close to the maximum of the probability distribution, and the tails of the distribution of a sum of random variables depends upon the distribution of the variables. Because the distribution of the matrices $m(\alpha)$ is non-universal, we therefore expect that the tail of the distribution of $\operatorname{tr} M_{N}$ is non-universal.

Further support for this prediction comes from considering moments of $M_{N}(\alpha)$. Modelling the $\alpha_{n}$ as random variables, we have

$$
\left\langle M_{N}(\alpha)\right\rangle=\prod_{n=1}^{N}\left\langle m\left(\alpha_{n}\right)\right\rangle=[\langle m(\alpha)\rangle]^{N}
$$

where the averages are taken over the invariant measure of the dynamics, which in the Hamiltonian case is just the uniform distribution on the phase-space area. Higher moments are obtained by averaging outer products of monodromy matrices: $\left\langle\operatorname{tr} M_{N}^{T}(\alpha) M_{N}(\alpha)\right\rangle$ for example may be expressed in terms of $[\langle m(\alpha) \otimes m(\alpha)\rangle]^{N}$ (see also [\$]). The value of $\left\langle\operatorname{tr} M_{N}^{T}(\alpha) M_{N}(\alpha)\right\rangle$ is therefore expected to grow exponentially with $N$

$$
\left\langle\operatorname{tr} M_{N}^{T}(\alpha) M_{N}(\alpha)\right\rangle \sim \exp \left(\lambda_{2} N\right)
$$

where $\lambda_{2}$ is the largest eigenvalue of $\langle m(\alpha) \otimes m(\alpha)\rangle$. A similar approach can be used to estimate the growth of $\left\langle\left(\operatorname{tr} M_{N}^{T} M_{N}\right)^{k}\right\rangle$ : the result is of the form (5) with $\lambda_{2}$ replaced by $\lambda_{2 k}$, the largest eigenvalue of the $k$-fold outer product $\langle m \otimes m \otimes \cdots \otimes m\rangle$. The values of these eigenvalues depend upon the structure of the elementary monodromy matrices $m$, indicating that the ratios of the eigenvalues $\lambda_{k}$ are non-universal. It is very difficult to reconcile this with the hypothesis that $P(N)$ is universal.

To test these predictions, the fraction of elliptic trajectories and the Lyapunov exponent were evaluated for the mappings defined by Eqs. (1) and (2). In Fig. 2 we plot the fraction $P(N)$ of elliptic trajectories as a function of $\lambda N$. Fig. 2 clearly shows the non-universality of $P(N)$.

Next we discuss the nature of the strong fluctuations apparent in Fig. 11. This figure indicates that the density of periodic points is highly non-uniform, however an exact result due to Hannay and Ozorio de Almeida [9] suggests that the periodic points might have a uniform distribution in phase space. In the following, we discuss how this apparent contradiction is resolved, and present an argument indicating that the density of periodic points has a log-normal distribution.

We base our discussion on the Kac-Rice [10,11] approach to calculating the densities of point singularities of random functions. The problem is to find the set of points where $\alpha=\mathcal{M}^{N}(\alpha)$ for large $N$. The basis of the Kac-Rice approach is to estimate the probability $\delta P$ of finding an example of the singularity in a small ball of volume $\delta V$ centred on an arbitrary test point: this can be written $\delta P \sim \mathcal{D} \delta V$, where $\mathcal{D}$ is the density of singularities. The estimate of $\delta P$ is usually simplified by the fact that the distance to a nearby singularity is given by a simple expression.

Assume that there is a periodic point at a small displacement $\delta \alpha$ from the test point $\alpha$. Considering the condition for periodicity $\alpha+\delta \alpha=\mathcal{M}(\alpha+\delta \alpha)$, and expanding the mapping to first order in $\delta \alpha$ leads to the approximation

$$
\delta \alpha=(M-I)^{-1}(\alpha-\mathcal{M})
$$

(where the index $N$ designating the $N$-th iterate has been dropped). For long orbits, it may be assumed that the joint distribution function $P[\mathcal{M}, M]$ factorizes, and

$$
\begin{aligned}
\mathcal{D} & =\left.P[\mathcal{M}]\right|_{\mathcal{M}=\alpha} \int d M P[M]|\operatorname{det}(M-I)| \\
& =\mathcal{A}^{-1}\langle|\operatorname{det}(M-I)|\rangle
\end{aligned}
$$

where $\mathcal{A}$ is the phase-space area. Similarly, the density of periodic points weighted with a smooth function $W(\alpha)$ is 


$$
\mathcal{D}_{W}=\mathcal{A}^{-1}\langle W(\alpha)|\operatorname{det}(M-I)|\rangle .
$$

Hannay and Ozorio de Almeida [9] considered the case $W(\alpha)=\left|w_{j}\right|^{2}$, where $w_{j}$ is the weight of the $j^{\text {th }}$ periodic orbit in the Gutzwiller sum. This case is of importance when estimating quantum two-point correlation functions semiclassically. The periodic orbit weights are of the form $w_{j}=\exp \left(\mathrm{i} \pi \mu_{j} / 2\right) \operatorname{det}(M-I)^{-1 / 2}$ where $\mu_{j}$ are integers termed Maslov indices which account for the phase changes associated with focusing or reflection. This gives $W(\alpha)=|\operatorname{det}(M-I)|^{-1}$, so that in this case $\mathcal{D}_{W}=\mathcal{A}^{-1}$, which constitutes the version of the sum rule derived in [9] which is applicable to maps. This simple, universal form is a result of the cancellation of the weight $|\operatorname{det}(M-I)|$ against its inverse.

We can now comment on the highly non-uniform distribution of periodic points shown in Fig. 1. It is natural to attempt to characterise this by a local density $\mathcal{D}(\alpha)$ defined in terms of the number of orbits inside a ball of radius, say, $\epsilon$. In order to construct a satisfactory definition using this approach, the local density would have to converge over a range of values of $\epsilon$, this range becoming broader as $N$ increases. The fluctuations in density are so wild that it appears to be impossible to define a local density in this way. Instead, we define the local density

$$
\mathcal{D}(\alpha)=|\delta \alpha|^{-d} .
$$

where $\delta \alpha$ is the displacement from the point $\alpha$ to the nearest periodic point, $|\delta \alpha|$ is the corresponding Euclidean distance, and $d$ is the phase-space dimension. For points sufficiently close to a periodic point, we have already seen that $|\delta \alpha| \sim\left|(M-I)^{-1}(\alpha-\mathcal{M}(\alpha))\right|$. The fluctuations of this quantity are dominated by those of $M$ which are log-normal. We plot the distribution of the local density evaluated according to (9) in Fig. 3. It is well described by a log-normal distribution.

We now discuss the implications of our findings for a minor variation upon a classical correlation function of periodic-orbit actions first introduced in [6]

$$
\begin{aligned}
C(T, \Delta S) & =\sum_{j \neq j^{\prime}} w_{j} w_{j^{\prime}}^{*} \delta_{\eta}\left(T-T_{j}\right) \\
& \times \delta_{\epsilon}\left(\Delta S-\left(S_{j}-S_{j^{\prime}}\right)\right) .
\end{aligned}
$$

Here, $S_{j}$ and $T_{j}$ are the action and period of the $j^{\text {th }}$ periodic orbit. In systems where Gutzwiller's trace formula is exact, and which exhibit universal spectral two-point correlations, $C(T, \Delta S)$ must be a function of the action difference which is universal within each of Dyson's symmetry classes [i]7]. In general, however, the trace formula cannot be exact. It is thus desirable to explore classical models for periodic-orbit correlations contained in (10).

A mechanism for such correlations is revealed by embedding the Hamiltonian (or mapping) into a oneparameter family. We remark that systems for which exact trace formulae exist (Riemann's zeroes, motion of a surface with constant negative curvature [4] and quantum graphs [12]) do not form one-parameter families exhibiting bifurcations. The parameter $X$ could be a coupling constant such as $K$ in Eq. (1). Varying the parameter $X$ produces bifurcations of orbits: immediately after a bifurcation the two orbits have the same actions $S_{j}=S_{j^{\prime}}$, and their weights $w_{j}$ and $w_{j^{\prime}}$ may be related in a simple way (typically they have opposite signs). We will estimate the contribution from bifurcations to the correlation function (10) at small values of $\Delta S$, as this can be directly related to the forms of the bifurcations. Assume that a bifurcation occurs at $X_{i}$ as a parameter $X$ is varied. The action difference in the neighbourhood of the bifurcation is of the form

$$
\left|\Delta S_{i}\right| \sim v_{i}\left|\Delta X_{i}\right|^{\beta}
$$

where $\Delta X_{i}=X-X_{i}$. The exponent $\beta$ will be determined from the type of bifurcation, and the constant $v_{i}$ depends on the particular bifurcation. The weights $w_{j}$ in (10) may be either singular or regular in the neighbourhood of the bifurcation: we allow for them having an algebraic singularity with exponents $\gamma_{j}$, which may be different for the two orbits in the vicinity of the bifurcation,

$$
w_{j} \propto\left|\Delta X_{i}\right|^{\gamma_{j}} .
$$

In the following we discuss a model for evaluating the contributions to (10) for long periodic orbits, treating the $w_{j}$ in (10) as random variables, which are independent except for those pairs of orbits which are related by a bifurcation:

$$
\left\langle w_{j}\right\rangle=0, \quad\left\langle w_{j} w_{j^{\prime}}\right\rangle=w^{2} \delta_{j j^{\prime}}
$$

where in the latter case it is assumed that the orbits $j$ and $j^{\prime}$ are not related by a bifurcation. At a bifurcation, one or both of the weights may be singular. In this case, it is reasonable to model the behaviour of $\left\langle w_{j} w_{j^{\prime}}\right\rangle$ as follows

$$
\left\langle w_{j} w_{j^{\prime}}\right\rangle \sim w^{2} \Lambda^{-\left(\gamma_{j}+\gamma_{j^{\prime}}\right)}|\Delta X|^{\gamma_{j}+\gamma_{j^{\prime}}}
$$

where $\Lambda$ characterizes the frequency with which bifurcations occur.

Let $\delta P_{\epsilon}(\Delta S)$ be the probability that a periodic orbit is connected to another orbit for which the action difference is in the interval $[\Delta S, \Delta S+\epsilon]$. According to the model above, the only contributions to the correlation function come from pairs of periodic orbits related by a bifurcation. The contribution from these orbits may be estimated as follows

$$
C \sim-\frac{1}{\epsilon}\left(\frac{d N}{d T}\right) w^{2}\left(\frac{\Delta X}{\Lambda}\right)^{\left(\gamma_{j}+\gamma_{j^{\prime}}\right)} \delta P_{\epsilon}(\Delta S)
$$

where $N(T)$ is the number of periodic orbits with period less than $T$, and $\Delta X$ is the typical distance to a bifurcation. From (11), the bifurcation occurs at a displacement in parameter space of the form $\Delta X \sim(\Delta S / v)^{1 / \beta}$. 
We model the probability that a periodic orbit does not undergo a bifurcation in a distance $\Delta X$ from an arbitrarily chosen test point as a Poisson distribution: this probability is $P_{ \pm}(\Delta X)=\exp \left[-\Lambda_{ \pm}|\Delta X|\right]$ for displacements to either side of the test point. For a sufficiently small separation $\Delta X$, the probability of finding a bifurcation in a small interval of size $\delta \Delta X$ on either side of $X_{0}$ is

$$
\delta P \sim \Lambda \delta \Delta X
$$

with $\Lambda=\Lambda_{+}+\Lambda_{-}$. Combining (11) and (16), one obtains $\delta P_{\epsilon}(\Delta S) \sim \Lambda v^{1 / \beta}|\Delta S|^{1 / \beta-1} \delta \Delta S$. Identifying $\delta \Delta S$ with $\epsilon$, and substituting into (15) produces the following result, valid for small $|\Delta S|$ :

$$
C(T, \Delta S) \propto|\Delta S|^{\left(1+\gamma_{j}+\gamma_{j^{\prime}}-\beta\right) / \beta} .
$$

Evaluation of the exponent in (17) requires information about the nature of the bifurcations. As an example, consider a hyperbolic billiard system with no corners, such as the Lorentz gas. In this case inverse bifurcations occur when pairs of similar orbits, only one of which bounces off a surface, become tangential to that scattering surface. Geometrical considerations imply that $\beta=2$, and $\gamma_{j}=0$ for the orbit which does not bounce tangentially but $\gamma_{j^{\prime}}=1$ for that which does. In this case the exponent in (17) is zero, but in other cases the exponent may be non-zero.

We summarise the conclusions on correlations of periodic orbits as follows. In systems with a smooth Hamiltonian, the statistic $P(N)$ is very closely related to the condition for bifurcations (namely that a periodic orbit intersects the manifold where $\operatorname{tr} M= \pm 2$ ). The statistic $P(N)$ was shown to be non-universal, and it is implausible that bifurcations will show universal behaviour in such systems. Totally chaotic systems have no elliptic trajectories, and bifurcations are associated with singularities of the Hamiltonian: in this case the arguments against universality are no less compelling. We therefore conclude that there is a non-universal component to the periodic orbit correlation function. Our result (17) also indicates that the contributions to the correlation function coming from bifurcations has a singularity if the exponent $1+\gamma_{j}+\gamma_{j^{\prime}}-\beta$ is non-zero. It is difficult to conceive of a non-universal and non-analtyic contribution to the correlation function which could combine with this one to give a universal result.

[1] F. Haake, Quantum signatures of chaos, Springer, Berlin (1992); O. Bohigas, in Disorder and Quantum Chaos, ed. J. Zinn-Justin, North-Holland, Amsterdam (1994);
Th. Guhr, A. Müller-Groeling and H.-A. Weidenmüller, Phys. Rep. 299 (1998) 190.

[2] F. J. Dyson, J. Math. Phys. 3 (1962) 1199-1215.

[3] B. V. Chirikov, Phys. Rep. 52 (1979) 263.

[4] M. Gutzwiller, Chaos in Classical and Quantum Mechanics, Springer, New York (1990).

[5] M. Wilkinson, J. Phys. A 21 (1988) 1173.

[6] N. Argaman, F-M. Dittes, E. Doron, J. P. Keating, A. Yu. Kitaev, M. Sieber, U. Smilansky, Phys. Rev. Lett. 71 (1993) 4326.

[7] U. Smilansky, D. Cohen and H. Primack, Ann. Phys (New York) 264 (1997) 108.

[8] A. Abrahams and M. J. Stephen, J. Phys. C 13 (1980) 377.

[9] J. H. Hannay and A. M. Ozorio de Almeida, J. Phys. A 17 (1984) 3429.

[10] M. Kac, Bull. Am. Math. Soc. 49 (1943) 314.

[11] S. O. Rice, Bell Sys. Tech. J. 24 (1945) 46.

[12] H. Schanz and U. Smilansky, chao-dyn/9904007.
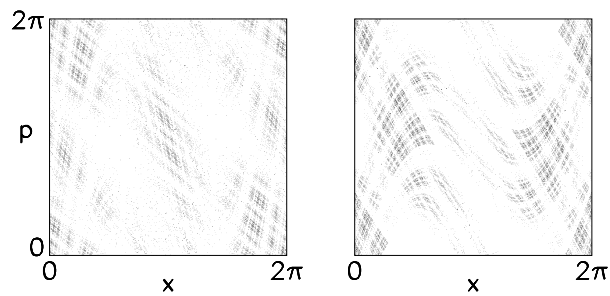

FIG. 1. Shows periodic points of the standard map with $K=6$ and $N=7$ (left) and for the modified cat map $K=2$ and $N=11$ (right). In both cases, $0 \leq x, p \leq 2 \pi$.

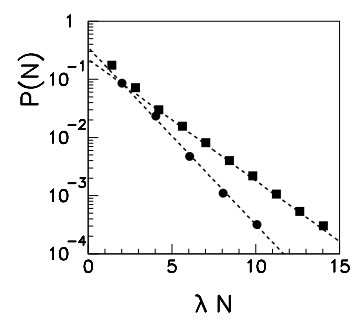

FIG. 2. Shows $P(N)$ as a function of $\lambda N$ for the standard map $(K=15, \lambda \simeq \log K / 2=2.015$, circles $)$ and for the modified cat map $(K=8, \lambda=1.404$, squares $)$.
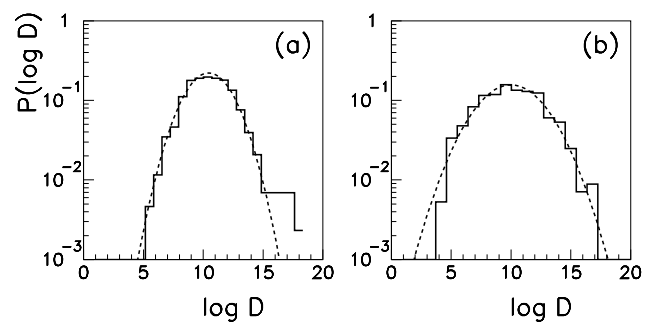

FIG. 3. Shows the distribution of the local density of periodic orbits $\mathcal{D}(\alpha)$ for the standard map with $K=6$ and $N=7$ (a) and for the modified cat map with $K=2$ and $N=11$ (b). In both cases the distribution is log-normal $(---)$. 